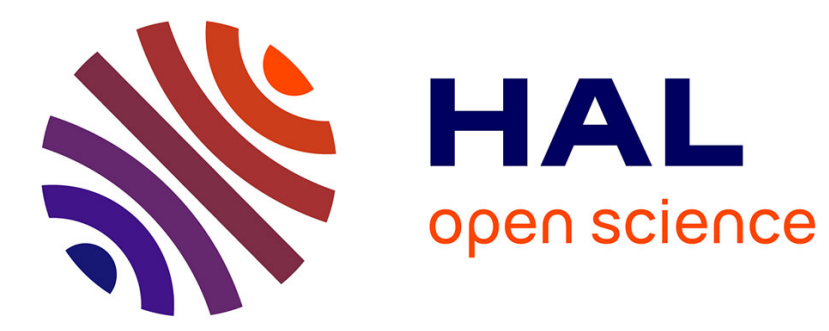

\title{
An Optimal Algorithm to Generate Extendable Self-Avoiding Walks in Arbitrary Dimension
}

\author{
Pascal Préa, Mathieu Rouault, François Brucker
}

\section{To cite this version:}

Pascal Préa, Mathieu Rouault, François Brucker. An Optimal Algorithm to Generate Extendable Self-Avoiding Walks in Arbitrary Dimension. Electronic Notes in Discrete Mathematics, 2017. hal01790908

\section{HAL Id: hal-01790908 \\ https://hal.science/hal-01790908}

Submitted on 14 May 2018

HAL is a multi-disciplinary open access archive for the deposit and dissemination of scientific research documents, whether they are published or not. The documents may come from teaching and research institutions in France or abroad, or from public or private research centers.
L'archive ouverte pluridisciplinaire HAL, est destinée au dépôt et à la diffusion de documents scientifiques de niveau recherche, publiés ou non, émanant des établissements d'enseignement et de recherche français ou étrangers, des laboratoires publics ou privés. 


\title{
An Optimal Algorithm to Generate Extendable Self-Avoiding Walks in Arbitrary Dimension
}

\author{
Pascal Préa ${ }^{1,2}$ \\ École Centrale Marseille \\ Laboratoire d'Informatique Fondamentale de Marseille \\ LIF, CNRS UMR 7279 \\ Marseille, France \\ Mathieu Rouault ${ }^{3}$ \\ University of Cape Town \\ Cape Town, Republic of South Africa \\ François Brucker ${ }^{4}$ \\ École Centrale Marseille \\ Laboratoire d'Informatique Fondamentale de Marseille \\ LIF, CNRS UMR 7279 \\ Marseille, France
}

\begin{abstract}
A self-avoiding walk (SAW) is extendable $[10,13]$ if it can be extended into an infinite SAW. We give a simple proof that, for every lattice, extendable SAWs admit the same connective constant that the general SAWs and we give an optimal linear algorithm to generate random extendable SAWs. Our algorithm can generate every extendable SAW in dimension 2. For dimension $d>2$, it generates only a subset of the extendable SAWs. We conjecture that this subset is "large" and has the same connective constant that the extendable SAWs. Our algorithm produces a kinetic distribution of the extendable SAWs, for which the critical exponent $\nu \approx .57$ for $d=2, .51$ for $d=3$ and .50 for $d=4,5,6$.
\end{abstract}

Keywords: Self Avoiding Walks, Connective Constant, Critical Exponent $\nu$, Random Generation. 


\section{Introduction}

A walk on a lattice is self-avoiding if it never passes twice through the same vertex (see Figure 1). Self-avoiding walks (SAWs) appeared as a model for polymers [7]. They also have applications in statistical physics [8] and in probability theory [15].
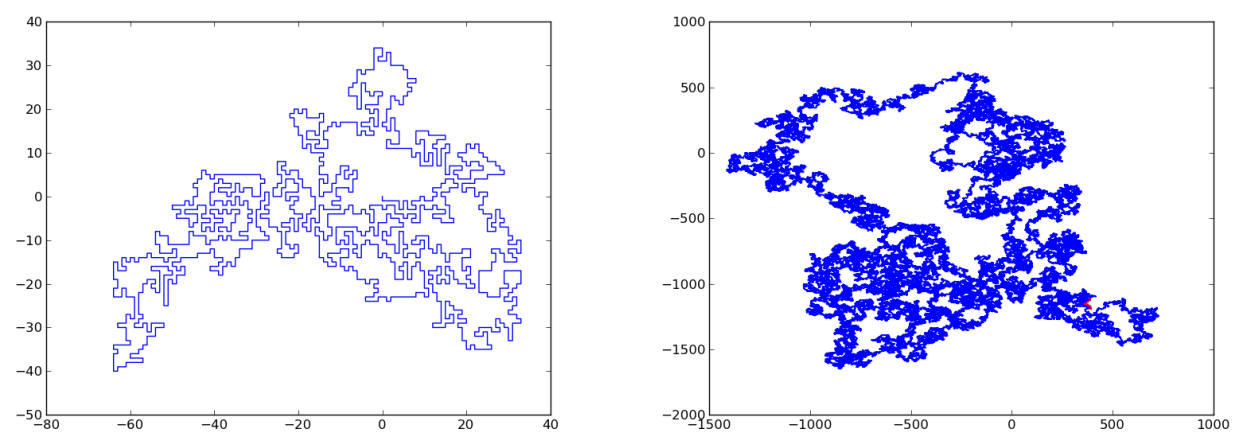

Fig. 1. A SAW of Length 1751 and a SAW of length $5 \cdot 10^{5}$ on the Square Lattice in Dimension 2

Formally speaking, for instance on the square grid $\mathbb{Z} \times \mathbb{Z}$ on plane, a walk is a sequence $W=\left(w_{0}, w_{1}, \ldots w_{n}\right)$ of vertices such that, for all $i<n, w_{i}$ and $w_{i+1}$ are neighbors (i.e. if $w_{i}=\left(x_{i}, y_{i}\right)$ and $w_{i+1}=\left(x_{i+1}, y_{i+1}\right)$, then $\left(x_{i+1}=x_{i}\right.$ and $\left.y_{i+1}=y_{i} \pm 1\right)$ or $\left(y_{i+1}=y_{i}\right.$ and $\left.\left.x_{i+1}=x_{i} \pm 1\right)\right)$. The walk $W$ is self-avoiding if $i \neq j \Rightarrow w_{i} \neq w_{j}$.

Despite a very simple and natural definition, and although they are very closed to random walks (which are standard objects, very well studied and known) self-avoiding walks ask many problems, both theoretical and practical.

Given a lattice (for instance, the hexagonal lattice on plane or the cubic one $\mathbb{Z} \times \mathbb{Z} \times \mathbb{Z}$ in space), the two main theoretical questions concerning SAWs on that lattice are:

- What is the number $c_{n}$ of SAWs of length $n$ ?

- Given a distribution on the SAWs (for instance the uniform distribution on the SAWs of length $n$ ), what is the average Euclidean distance $d_{n}$ between

$\overline{1}$ Part of this work was done while one of the authors (P.P.) was visiting the University of Cape Town.

2 Email: pascal.prea@lif.univ-mrs.fr

3 Email: mathieu.rouault@uct.ac.za

4 Email: francois.brucker@lif.univ-mrs.fr 
the two extremities of a SAW of length $n$ ?

It is conjectured [15] that:

- $c_{n} \sim \mu^{n} n^{\gamma-1}$

- $d_{n} \sim n^{\nu}$

It is strongly believed that the critical exponents $\gamma$ and $\nu$ depend only on the dimension (and not on the lattice) while the connective constant $\mu$ also depends on the lattice. It is known that the connective constant (which is equal to $\lim _{n \rightarrow \infty} c_{n}^{1 / n}$ ) exists for every lattice. Despite decades of efforts, these questions remain unsolved, especially in low dimensions. It has recently been shown [6] that the connective constant for the hexagonal lattice on plane is $\sqrt{2+\sqrt{2}}$. Conversely, for the square lattice on plane, it is conjectured [11] that the connective constant is the unique positive root of $13 x^{4}-7 x^{2}-581=0$ (i.e. $\sqrt{\frac{7+\sqrt{30261}}{26}} \approx 2.64$ ).

For two-dimensional lattices, it is conjectured that $\gamma=43 / 32$ and that, for the uniform distribution, $\nu=3 / 4$.

From a practical point of view, it is very interesting to generate random self-avoiding walks since these walks arise as models for various physical phenomena. In addition, generating random self avoiding walks yields an approximation to the critical exponents and the connective constant. There are many algorithms to generate random self-avoiding walks; among them the pivot algorithm [14,12], the Berretti-Sokal algorithm [1], the Rosenbluth algorithm $[19,9,18]$, flat-PERM [5]. All these algorithms are Monte Carlo algorithms $[17]$.

The (apparently) simplest way to generate a random SAW consists by choosing, at each step, a neighbor of the courant end of the walk, not already in the walk and to add it to the walk. When the last point of the walk has no "free neighbors", we can stop or use backtracking. Backtracking needs prohibitive computer ressources, and, dimension 2, not using it yields only short paths (see Figure 2).

In this paper, we study a special case of self-avoiding walks : the extendable $S A W s[10,13]$. In Section 2, we give their definition and some properties, and in Section 3, we give an optimal $O(n)$ algorithm to generate a random extendable SAW of length $n$. An implementation of this algorithm can be found at https://github.com/pascalprea/Extendable_SAW. 

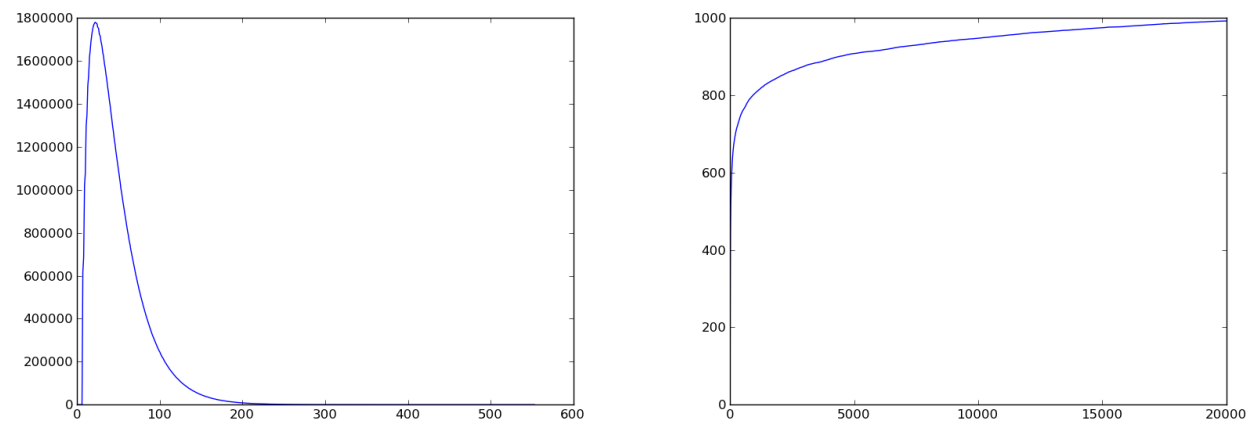

Fig. 2. Histogram of Length Without Using Backtracking (left) and by Using Backtracking (Right) for SAWs on the planar lattice $\mathbb{Z} \times \mathbb{Z}$. The left histogram was obtained with $10^{8}$ trials. On the $x$-axis are the lengths, and on $y$-axis the number of SAWs having these lengths. The right histogram was obtained with $10^{5}$ trials. On the $x$-axis is the number of steps ( $\approx$ the time), with scale $1 / 100$ and on the $y$-axis the average length at this moment.

\section{Definitions and Properties}

We say that a SAW $W=\left(w_{0}, w_{1}, \ldots w_{n}\right)$ is extendable if $W$ can be extended into an infinite SAW (i.e. if there exists an infinite SAW $W_{\infty}=$ $\left.\left(w_{0}, w_{1}, \ldots w_{n}, \ldots\right)\right)$.

Proposition 2.1 For every regular lattice of the Euclidean space (e.g. the triangular lattice in the plane or the cubic lattice in the 3-dimensional space), extendable SAWs admit a connective constant which has same value that the connective constant for general SAWs.

This result is a particular case of Theorem 1 in [10]; it admits a very simple proof that we give here.

Proof. We first show that extendable SAWs admit a connective constant $\mu_{e}$. The proof is exactly the same that the one for general SAWs (see [15] p. 910); we will only give its sketch. Let $c_{n}^{e}$ be the number of extendable SAWs of length $n$. Every extendable SAW of length $n+m$ is the concatenation of an extendable SAW of length $n$ and of an extendable SAW of length $m$. So, $c_{n+m}^{e} \leq c_{n}^{e} \times c_{m}^{e}$, thus $\log c_{n+m}^{e} \leq \log c_{n}^{e}+\log c_{m}^{e}$ and $\mu_{e}=\lim _{n \rightarrow \infty}\left(c_{n}^{e}\right)^{1 / n}$ exists.

We now prove that $\mu_{e}=\mu$. Let $W=\left(w_{1}, w_{2}, \ldots, w_{n}\right)$ be a SAW of length $n, W^{\star}=\left(w_{1}, w_{2}, \ldots w_{\lfloor\sqrt[3]{n}\rfloor}\right)$ is an extendable SAW (otherwise, the SAW $W^{+}=\left(w_{\lfloor\sqrt[3]{n}\rfloor}, \ldots, w_{n}\right)$ would be entirely included in a finite volume whose surface is $W^{\star}$; but if the dimension of the space is $>1$, a volume has 
a surface greater than its cubic root). Thus $c_{n} \leq c_{\sqrt[3]{n}}^{e} \cdot c_{n-\sqrt[3]{n}}$; so, for $n$ large enough, $\mu^{n} \leq \mu_{e}^{\sqrt[3]{n}} \cdot \mu^{n-\sqrt[3]{n}}$ and $\mu_{e} \geq \mu$. As $\mu_{e} \leq \mu$, the property is proved.

Given an extendable SAW $W=\left(w_{0}, w_{1}, \ldots w_{n}\right)$, an Ariadne thread (associated with $W)$ is an infinite SAW $A=\left(a_{0}, a_{1}, \ldots\right)$ such that:

- $a_{0}$ and $w_{n}$ are neighbors.

- $A$ and $W$ do not cross $\left(\forall i, j, w_{i} \neq a_{j}\right)$

Equivalently, the concatenation (of $W$ and $A) W A=\left(w_{0}, w_{1}, \ldots, w_{n}, a_{0}, a_{1}, \ldots\right.$ ) is an infinite SAW; so, a self-avoiding walk is extendable if and only if it admits an Ariadne thread.

\section{An Optimal Algorithm to Generate Random Extend- able Walks}

\subsection{Description of the algorithm}

Our algorithm constructs a SAW step by step, starting with the empty walk and adding a point at each step. Its main idea consists in maintaining, at each step, an Ariadne thread. We will describe it for the hypercubic lattice $\mathbb{Z}^{d}$.

If $n=0$, every axis-parallel half line starting at $w_{0}$ is an Ariadne thread for $W$. If $n>0$, the SAW $W_{n-1}=\left(w_{0}, w_{1}, \ldots w_{n-1}\right)$ admits an Ariadne thread $A_{n-1}=\left(a_{0}, a_{1}, \ldots\right)$. The construction/modification of the Ariadne thread will depend on the configuration at the last point $w_{n}$ of $W$ (we suppose that $W$ is an extendable SAW, and so $\left.w_{n} \notin W_{n-1}\right)$ :

(i) There exists a axis-parallel half line $L=\left(a_{0}=w_{n}, a_{1}, \ldots\right)$ which do not cross $W_{n-1}$ (we say that $w_{n}$ is sees the infinite). In this case, $A=$ $\left(a_{1}, a_{2}, \ldots\right)$ is an Ariadne thread for $W$.

(ii) The point $w_{n}$ is on $A_{n-1}$, i.e. there exists $i$ such that $w_{n}=a_{i}$. In this case, $A=\left(a_{i+1}, a_{i+2}, \ldots\right)$ is an Ariadne thread for $W$.

(iii) The point $w_{n}$ does not see the infinite and is not on $A_{n-1}$. There exists a path $P$ avoiding $W_{n-1}$ between $w_{n}$ and $A_{n-1}$. For algorithmic efficiency, we only search this path in a neighborhood of $w_{n-1}$ (namely the points at $L_{\infty}$-distance 1 from $w_{n-1}$ ). The concatenation of $P$ and (the end of) $A_{n-1}$ is an Ariadne thread for $W$.

Proposition 3.1 Every extendable 2-dimensional SAW can be generated by our algorithm.

Proof. We only have to prove that, at point 3 of the algorithm, the path $P$ contains points at $L_{\infty}$-distance 1 from $w_{n-1}$. 
In this case, up to symmetries and rotations, we are in one of the cases of Figure 3. Since $W$ is extendable, in all cases, the points marked with a white circle cannot be on $W_{n-1}$ : if a point $w_{i}(i<n-1)$ is one of these points, $\left(w_{i}, \ldots, w_{n-2}, w_{n-1}\right)$ is a loop which separates the plane into two connected components, one containing $w_{n}$ and the other $a_{0}$. By Jordan's Theorem, one of these two components is finite, which is impossible since both $a_{0}$ and $w_{n}$ are "linked to the infinite" by a walk which does not cross $W_{n-1}$.
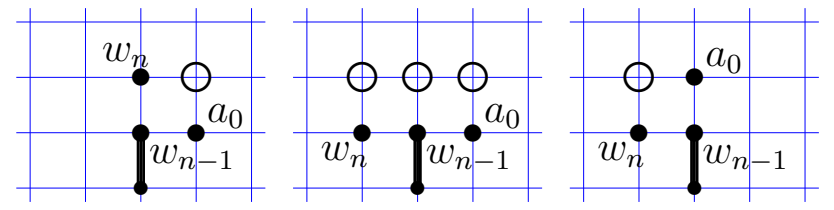

Fig. 3. The three cases with point $w_{n}$ which does not see the infinite and is not on $A_{n-1}$.

So, by adding (in the right order) the point marked with a white circle at the beginning of $A_{n-1}$, we get an Ariadne thread for $W$ (it is possible that one of these white points is already in $A_{n-1}$, in this case, we only have to suppress the loop inside $A$ ).

Proposition 3.2 If we assume that the basic operations on a hash table (searching, adding or deleting an element) run in time $O(1)$, then adding a new point to an extendable $S A W$ runs in time $O(1)$. Thus, generating a extendable $S A W$ of length $n$ in $\mathbb{Z}^{d}$ takes $O(n)$ time.

Proof. We represent the walk $W$ by a list $W^{L}$ (which will be the output) and $d+1$ associative arrays:

- $W_{P}$ whose keys are the points.

- For each coordinate $i, W_{i}$ whose keys are the $d-1$ other coordinates of the points.

$W_{i}\left[\left(x_{1}, x_{2}, \ldots x_{i-1}, x_{i+1}, \ldots, x_{d}\right)\right]=\left[y_{\min }, y_{\max }\right]$, where $y_{\min }\left(\operatorname{resp} . y_{\max }\right)$ is the smallest (resp. greatest) $i$ th coordinate of points in $W$ having $x_{1}, \ldots x_{i-1}$, $x_{i+1}, \ldots, x_{d}$ as other coordinates.

When we create a new thread (i.e. when we add to the walk a point that see the infinite), we only store the starting point of the half-line. We represent the Ariadne thread $A$ by a (finite) list $A^{L}$ (the first element of $A^{L}$ is the oldest in the thread) and an associative array $A_{P}$. The keys of $A_{P}$ are the points of $A$; if $a$ is a point of $A, A_{P}[a]$ is the index of $a$ in $A^{L}$.

In order to add a new point to $W$, we consider (in random order) the neighbors of the last point $w_{n-1}$ of $W$. Let $w=\left(x_{1}, \ldots x_{d}\right)$ be one of these 
neighbors, we (in this order):

(i) Check if $w \in W$.

(ii) Check if $w$ sees the infinite (and modify $W$ and $A$ if it is the case).

(iii) Check if $w \in A$.

(iv) Check if there is a path not crossing $W$ between $w$ and $A$ included in the neighborood of $w_{n-1}$.

In order to check if $w \in W$, we just have to test if $w$ is a key of $W_{p}$. This takes $O(1)$.

To check if $w$ sees the infinite, we have to compare each coordinate $x_{i}$ with the values $y_{\min }$ and $y_{\max }$ stored in $W_{i}\left[\left(x_{1}, \ldots, x_{i-1}, x_{i+1}, \ldots, x_{d}\right)\right]$ ( $w$ sees the infinite if and only if $\exists i: x_{i}<y_{\min }$ or $\left.x_{i}>y_{\max }\right)$. This test takes $O(1)$. If $w$ sees the infinite the, we have to add it in $W^{L}$ and $W_{p}$; we also have to modify the $W_{i}$ (if $x_{i}<y_{\min }, y_{\min } \leftarrow x_{i}$ and if $x_{i}>y_{\max }, y_{\max } \leftarrow x_{i}$ ) and to create $A^{L}$ and $A_{P}$ with one point inside. All this runs in time $O(1)$.

Checking if $w \in A$ takes $O(1)$. If it is the case, we have to add $w$ to $W$ and also to delete all points of $A$ which have been added (in $A$ ) after $w$, i.e. all the elements of $A^{L}$ with index $\geq A_{P}[(x, y)]$ (with all the corresponding elements of $\left.A_{P}\right)$. Deleting one point takes $O(1)$, but it is possible that, at one step, many points of $A$ have to be deleted. At each step, we add at most $2^{d}-1$ points in $A$. So, between the beginning of the generation of $W$ and Step $n$, it is impossible to delete more than $\left(2^{d}-1\right) n$ elements of $A$. The amortized complexity of this step is thus $O(1)$.

For the last test, we only have to find a path in a graph with at most $2^{d}-1$ vertices and to add a point to $W$ and a bounded number of points to $A$. This takes $O(1)$. We may have to delete a loop in $A$, but as for the precedent case, the amortized complexity of this deletion is $O(1)$.

Proposition 3.3 Generating a extendable $S A W$ of length $n$ in $\mathbb{Z}^{2}$ takes $O(n)$ time.

Proof. It is sufficient to replace, in the proof of Proposition 3.2, the associative arrays $W_{P}, W_{i}$ (for $\left.i=1,2\right)$ and $A_{P}$ by data structures whose access time is in $O(1)$ in worst case. The associative arrays $W_{P}$ and $A_{P}$ can be replaced by the data structure of [4], as adapted to $\mathbb{Z} \times \mathbb{Z}$ in [2]. Each $W_{i}$ can be replaced by two lists (one for the positive coordinates, and the other for the negative ones). Since the paths start from the origin, if a point $(x, y)$ is in a path, there will be a point with abscissa $x-1$ if $x>0$ or $x+1$ if $x<0$ (and similarly for ordinate $y$ ). So these lists will have no unused cases. 
Remark: It is possible (for the general algorithm in $\mathbb{Z}^{n}$ ) to use balanced search trees instead of associative arrays. In this case, the complexity of adding a point to an extendable SAW is $O(\log n)$ and so, the complexity of generating a extendable SAW of length $n$ is $O(n \log n)$.

In dimension 2 , our algorithm is very close to the one in $[3,5]$ which relies on a "winding number" that cannot be generalized in higher dimension. The advantage of our algorithm is that it works in any dimension. In dimension $>2$, our algorithm generates a subset of the extendable SAWs. We conjecture that the set of the SAWs generated by our algorithm is large enough to admit a connective constant equal to the one for general SAWs (hints for this results are given by the fact that extendable SAWs that cannot be generated by our algorithm have to contain very particular configurations and that for dimension 3 and larger the estimated critical exponent $\nu$ of our extendable SAWs equals the one obtained for random walks (see below)).

\subsection{Tests and variants}

Since the algorithm is linear, it is possible to generate long SAWs in rather short time. On table 1 are shown results of tests made in Python 2.7 on an Intel Core i5 at $2.7 \mathrm{GHz}$. Although these tests were made in $\mathbb{Z} \times \mathbb{Z}$, we used the algorithm with associative arrays. It is possible to generate, in approximatively 12 seconds, an extendable SAW of length $10^{6}$. We obtain SAWs like those in Figure 4.
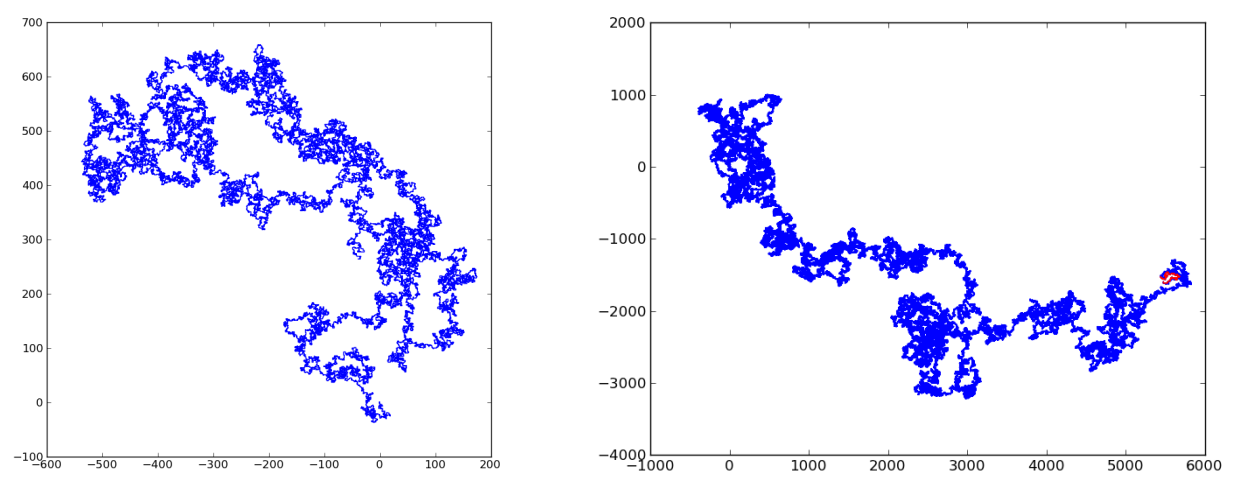

Fig. 4. Two SAWs on $\mathbb{Z} \times \mathbb{Z}$ obtained with our algorithm. One of length 50000 and one of length $10^{6}$.

We have measured the average distance between the two extremities of extendable SAWs. We have made 20000 trials on SAWs of length 100, 200, 


\begin{tabular}{|r|r|r|}
\hline length & $\begin{array}{r}\text { total time for } \\
100 \text { trials }(\mathrm{s})\end{array}$ & $\begin{array}{r}\text { average } \\
\text { time }\end{array}$ \\
\hline \hline 1000 & 0.920 & $9 \mathrm{~ms}$ \\
5000 & 4.885 & $49 \mathrm{~ms}$ \\
10000 & 9.879 & $99 \mathrm{~ms}$ \\
50000 & 52.569 & $526 \mathrm{~ms}$ \\
100000 & 106.056 & $1.06 \mathrm{~s}$ \\
500000 & 570.968 & $5.7 \mathrm{~s}$ \\
1000000 & 1175.337 & $11.8 \mathrm{~s}$ \\
\hline
\end{tabular}

Table 1

Time taken by the generation of extendable SAWs.

$\ldots, 900,1000,2000, \ldots, 10000,20000, \ldots$ until $10^{6}$. The results are shown in Figure 5.
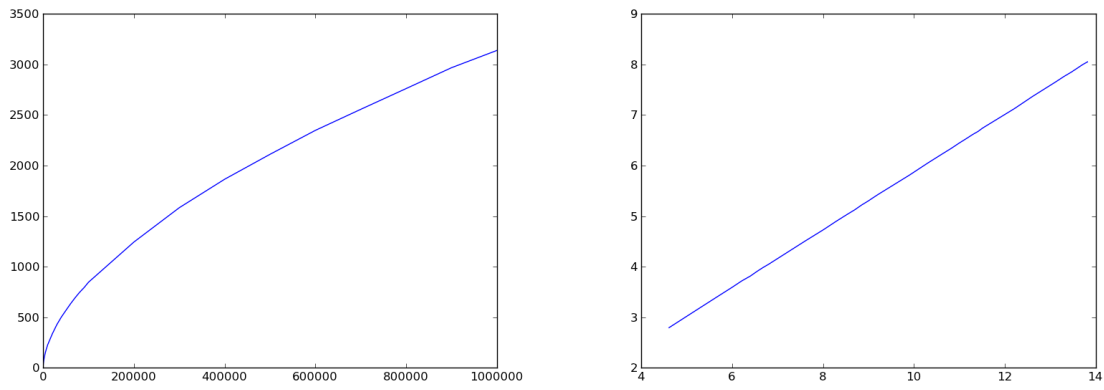

Fig. 5. Average distance between the two extremities of extendable SAWs on $\mathbb{Z} \times \mathbb{Z}$, for lengths in $\left\{100,200, \ldots, 1000,2000, \ldots, 10^{4}, \ldots 10^{6}\right\}$. The left curve represents the average distance as a function of the length. The right curve represents the log of the average distance as a function of the log of the length.

The slope of the straight line of Figure 5 is 0.571 , and its correlation coeficient is $>0.999$. From these tests, we got .57 as an estimated value for the critical exponent $\nu$ for the lattice $\mathbb{Z} \times \mathbb{Z}$, which confirms the value got in [13] by enumeration of all the extendable SAWs of length $\leq 22$. This value is different from the one expected for the uniform distribution (.75); actually, our algorithm generates a kinetic distribution [16] of extendable SAWs.

Similarly, we got $\nu \approx .51$ for the lattice $\mathbb{Z}^{3}$ and $\nu \approx .50$ for $\mathbb{Z}^{4}, \mathbb{Z}^{5}$ and $\mathbb{Z}^{6}$. These results are coherent with the fact that in high dimensional space, SAW 
and random walks are quite similar.

We can change the distribution of SAWs generated by our algorithm in the following way: on the lattice $\mathbb{Z} \times \mathbb{Z}$, let $x_{1}, \ldots x_{4}$ be the neighbors of the last point of the SAW. If it is possible to extend $W$ with $x_{i}$, we define $p_{i}=K+h_{i}$, where $h_{i}$ is the number of half-lines starting at $x_{i}$ and not crossing $W$, and $K$ is a fixed parameter. If it is not possible to extend $W$ with $x_{i}, p_{i}=0$. Then we choose $x_{i}$ with probability $p_{i} / \sum_{j=1}^{4} p_{j}$. Such a modification disadvantages dense SAWs. On the contrary, by choosing $p_{i}=K+4-h_{i}$, we favor dense SAWs. In Figure 6, one can see SAWs that we get, with different corrections. These different appearances correspond with different values of the critical exponent $\nu$ (see Figure 8).
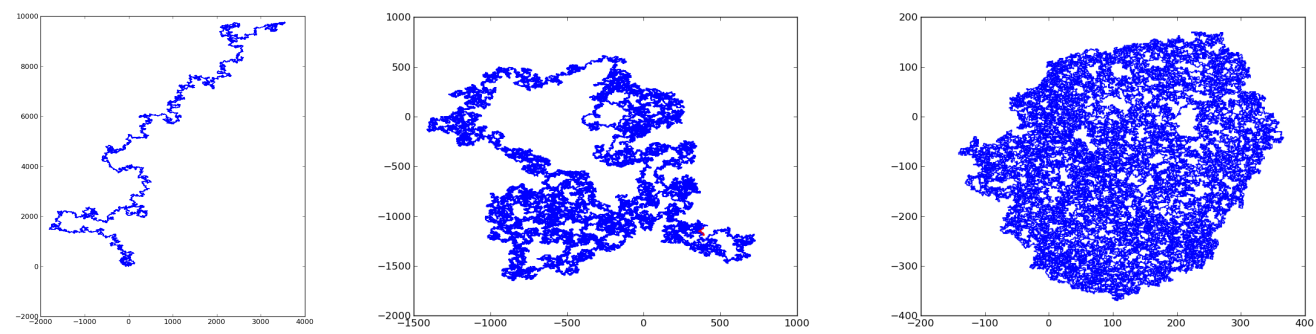

Fig. 6. 2-dimensional SAWs of length $5 \cdot 10^{5}$, with correction $p_{i}=7+h_{i}$, with no correction and with correction $p_{i}=4-h_{i}$ (from left to right).

We have chosen a "local" correction because we want to avoid backtracking. So this variant of our algorithm is also linear in time; it takes approximatively twice the time of the original one. Another (a posteriori) reason is that "it works", in the sense that, for every value of $K$, we can get a value for the critical exponent $\nu$. As for the basic algorithm, this value is the slope of a straight line (the log of the average distance as a function of the log of the length) and, in each case, the correlation coefficient is $>0.997$.

\subsection{Adaptation of the algorithm to other tilings}

The definition of the Ariadne thread does not depend on the tiling. The only configuration that changes is the one were the last point of the walk does not see the infinite and is not on the thread. In Figure 7 are shown some cases for the hexagonal and the triangular lattice of the plane. 

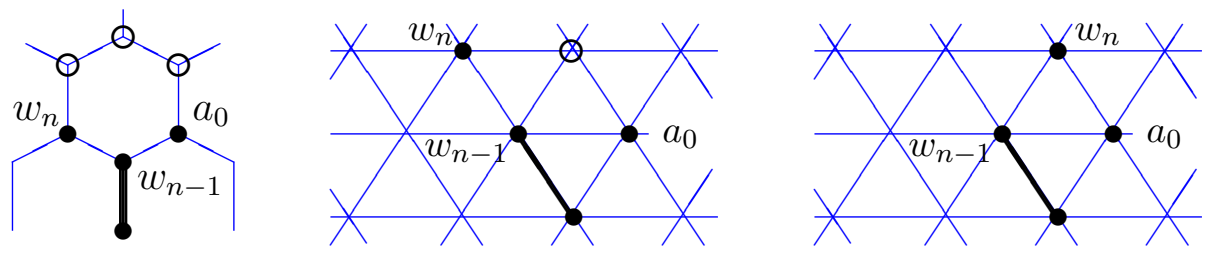

Fig. 7. Configurations where the last point of an extendable SAW does not see the infinite and is not on the Ariadne thread for the hexagonal and the triangular lattices. For the triangular lattice, it is possible that $w_{n}$ is a neighbor of $a_{0}$ (on the right of the figure); there is only one such configuration (up to rotations and symmetries). There are three configurations like the one of the middle of the figure, where $w_{n}$ is not a neighbor of $a_{0}$. Notice that, in all cases, $a_{0}$ is a neighbor of the two last points of the walk. For the hexagonal lattice, there is only one configuration.

\section{Conclusion}

We have presented in this paper a linear, and thus optimal, algorithm to generate random self avoiding walks on the lattice $\mathbb{Z}^{d}$. This algorithm generates extendable SAWs, and these SAWs have the same connective constant that the general SAWs. Our algorithm produces a kinetic distribution on the extendable SAWs for which the critical exponent $\nu$ is $\approx 0.57$ in dimension 2 (.51 if $d=3$ and .50 for $d=4,5$ or 6 ). We have also presented a variant of our algorithm, also linear, which depends on a parameter $K$. For any $x \in[0.49, \ldots, 0.966]$, it is possible to determine a value of $K$ such that our algorithm gives a distribution of self-avoiding walks with critical exponent $\nu=x$.

Our algorithm can easily be adapted to other lattices like the Archimedean lattices of the plane since, in every tiling of the plane, the construction /modification of an Ariadne thread yields to the three same cases. It is also possible to "prune-enrich" it [9].

\section{References}

[1] Berretti, A. and A.D. Sokal, New Monte Carlo Methods for the Self-Avoiding Walk, J. Stat. Phys. 40 (1985), 483-531.

[2] Blondin Massé, A. and É. Marcotte, A generic data structure for representing discrete paths on regular grids, GASCom 2016, Bastia.

[3] Bousquet-Mélou, M., On the Importance Sampling of Self-Avoiding Walks, Combinatorics, Probability and Computing 23(5) (2014), 725-748. 


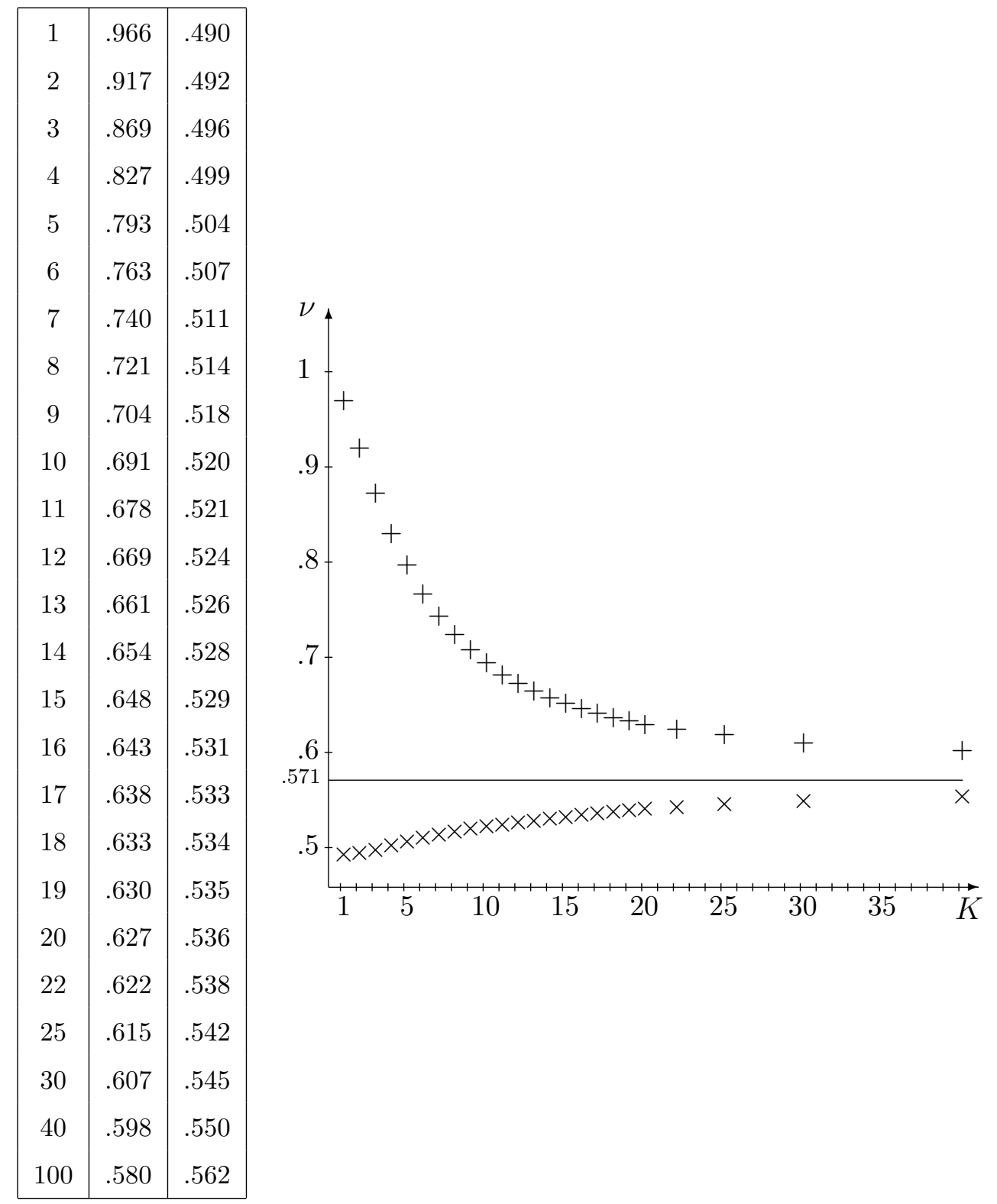

Fig. 8. Critical Exponent $\nu$ depending on the parameter $K$. In the table, $K$ is in the first column, $\nu$ for correction $p_{i}=K+h_{i}$ is in the second column, and $\nu$ for correction $p_{i}=4+K-h_{i}$ is in the third columns. The $x$-axis of the figure represents $K$, and $\nu$ is on the $y$-axis. The values for $p_{i}=K+h_{i}$ are marked with + , and those for $p_{i}=4+K-h_{i}$ with $\times$. All these values have been estimated with 2000 trials on SAWs of length $100,200, \ldots, 900,1000,2000 \ldots 9000,10000, \ldots 10^{6}$. In all cases, the correlation coefficient of the log-log line is $>.997$. 
[4] Brlek, S., M. Koskas and X. Provençal, A linear time and space algorithm for detecting path intersections, Theoretical Computer Science 412 (2011), 48414850 .

[5] Chan, Y.B. and A. Rechnitzer, A Monte-Carlo Study of Non-Trapped SelfAvoiding Walks, J. Phys. A: Math. Theor. 45 (2012), 405004.

[6] Duminil-Copin, H. and S. Smirnov, The Connective Constant of the Honeycomb Lattice Equals $\sqrt{2+\sqrt{2}}$, Annals of Mathematics 175(3) (2012), 1653-1665.

[7] Flory, P.J., The Configuration of a Real Polymer Chain, J. Chem. Phys. 17 (1949), 303-310.

[8] Gennes, P.G. de, Exponents for the Excluded Volume Problem as Derived by the Wilson Method, Phys. Lett. A 38 (1972), 339-340.

[9] Grassberger, P., Pruned-Enriched Rosenbluth Method: Simulation of $\theta$-Polymers of Chain Length up to 1000000, Phys. Rev. E 56 (1997), 3682-3693.

[10] Grimmett, G.R., A.E. Holroyd and Y. Peres, Extendable Self-Avoiding Walks, Annales de l'Institut Henri Poincaré D, 1 (2014), 61-75.

[11] Jensen, I. and A.J. Guttmann, Self-Avoiding Polygons on the Square Lattice, J. Phys. A 32 (1999), 4867-4876.

[12] Kennedy, T., A Faster Implementation of the Pivot Algorithm for Self-Avoiding Walks, J. Stat. Phys. 106 (2002), 407-429.

[13] Kremer, K. and J.W. Lyklema, Indefinitely growing self-avoiding walk, Phys. Rev. Lett., 54 (1985), 267-269.

[14] Lal, M., Monte Carlo Computer Simulation of Chain Molecules, J. Mol. Phys. 17 (1969), 57-64.

[15] Madras, N. and G. Slade, "The Self-Avoiding Walk", Birkhäuser, 1993.

[16] Majid, I., N. Jan, A. Coniglio and H.E. Stanley, Kinetic Growth Walk: a New Model for Linear Polymers, Phys. Rev. Lett. 52 (1984), 1257-1260.

[17] Metropolis, N. and S. Ulam, The Monte-Carlo Method, J. Am. Stat. Ass. 44 (1949), 335-341.

[18] Rechnitzer, A. and E.J. Janse van Rensburg, Generalized Atmospheric Rosenbluth Methods (GARM), J. Phys. A 41 (2008), 442002-442010.

[19] Rosenbluth, M.N. and A.W. Rosenbluth, Monte Carlo Calculations of the Average Extension of Molecular Chains, J. Chem. Phys. 36 (1955), 356-359. 